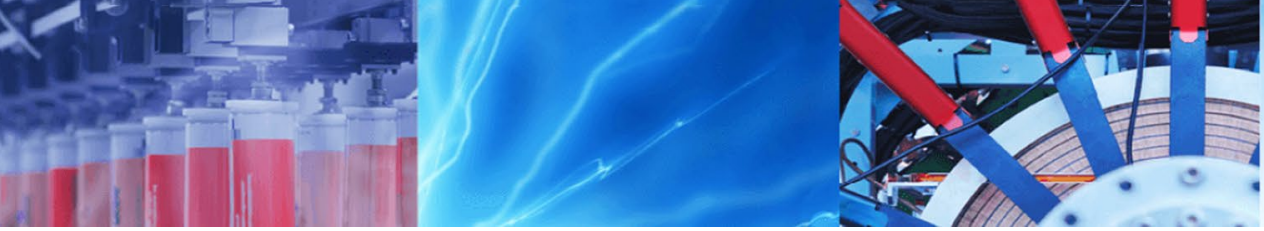

Research Article

\title{
Numerical analysis and application of electric field grading device for metal-enclosed switchgear
}

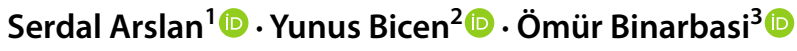

Received: 7 September 2020 / Accepted: 21 January 2021 / Published online: 26 January 2021

(C) The Author(s) 2021 OPEN

\begin{abstract}
Electric field grading devices have great importance for both the electric power transmission and distribution systems. This paper presents an improved electric field grading device used in the medium voltage metal-enclosed switchgear. The solutions have been carried out by using Ansys Maxwell 3D software. The structure of two electrodes designed as cylindrical and elliptical have been compared to determine the electric field grading device to be used in practice. The transient and steady-state analyses have been performed in both designs. The field grading device manufactured for the metal-enclosed switchgear has been tested according to IEC standards in a high voltage laboratory environment. This study revealed that the newly designed field-grading device can be used in the metal-enclosed switchgear more safely. It also encourages researchers to optimize the electric field distribution using different geometric structures and to create running conditions in smaller indoor environments.
\end{abstract}

Keywords Electric field · Electric transient · Grading · Numerical analysis · Switchgear

\section{Introduction}

In power systems, electric field grading devices used both to extend the life of the designed equipment and to reduce the number of failures have great importance. These devices minimize the probability of failure caused by abnormal situations such as dielectric breakdown, arcing, electrical discharge, etc. [1-4]. Metal-enclosed switchgear is a typical electrical system exposed to this type of failure in the electric power system [5] and in order to protect the insulator-metal junctions, electric field grading devices can be used $[6,7]$. Manufacturers are required to produce quality products within the framework of standards or at the request of the customer. Also, manufacturers should consider the costs of production, while fulfilling this request. For this purpose, designers reduce the weight and size of the electrical equipment using optimization techniques in order to meet the requirements of the system [8]. Electric field computation procedures are an important stage of these actions. Various numerical techniques can be used for computing the electric fields $[4,9]$. Finite element method (FEM) is the best known numerical technique which enables the designer to solve the difficult problem such as non-uniform surface and to make a practical electrical design optimization [8-11].

In the literature, there are few studies regarding both theory and practice in electric field grading. Some of these studies were conducted to make optimization of the high voltage system in outdoor substations and this type studies focus on the environmental conditions (such as dry-wet condition, moisture, pollution, etc.) to determine electric field stress and to optimize the electric field grading devices [10,12-15]. Some studies were conducted to make optimization of the indoor high and medium voltage

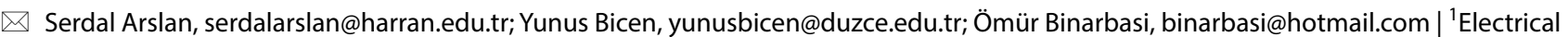
Department, Birecik Vocational School, Harran University, Sanliurfa, Turkey. ${ }^{2}$ Department of Electrical-Electronics Engineering, Duzce University, 81620 Duzce, Turkey. ${ }^{3}$ The Turkish Electromechanical Industry Company, 06200 Yenimahalle, Ankara, Turkey. 
systems and they focus on cabinet size optimization and system safety, in addition to the other electrical aspects [5, $16,17]$. Some of the numerical studies were carried out on the electric field distribution for transient voltages $[18,19]$. However electric field grading devices in metal-enclosed switchgear and impulse effects have been not considered sufficiently. These kinds of studies are more complicated and difficult. Because many factors such as panel walls, corner points, connectors, etc. that could affect the electric field distribution of the system should be considered [16, $17,20]$.

The motivation of this study is to manufacture a new electric field grading device with an original design to be used in metal-enclosed switchgear units. The contributions of the study can be written as follows: (a) placing the switchgear equipment in a smaller volume than before, (b) reducing the possibility of electrical arcs, (c) reducing production and maintenance costs, (d) ensuring operational continuity.

In this context, the article is organized as follows: Sect. 2 presents the numerical technique features. In the third chapter, technical details are presented for two different devices designed to grading the electric field in the switchgear. In this section, both steady state and transient state analyzes have been carried out by utilizing the FEM in terms of design features. In the fourth part, it has been checked whether the system meets IEC standards [21, 22] namely the power frequency voltage withstand and the impulse voltage test, in an accredited high voltage laboratory. Finally, conclusions are summarized in Sect. 5 .

\section{Numerical technique}

Uniform models and simplified assumptions can be used to reach an approximate analytic solution for simple systems, while they are not suitable for the correct solution of complex models as in this study [23,24]. Different methods such as FEM, boundary element method, and the finite difference method can be used to solve complex electric field problems that cannot be solved easily using analytical techniques [9]. Various commercial software packages that use these methods are available. Appropriate software should be chosen considering some features such as solution speed, the accuracy of calculation, the memory requirement for the solution, ease of use. In this regard, the selected software Ansys-Maxwell has many capabilities such as having a large drawing infrastructure, more solution options, providing a dynamic analysis of the designed model, etc.

In this study, the electrostatic analysis and the electric transient analysis have been performed using FEM. Unlike an electrostatic solution, the electric transient voltage curve is defined into the design data sets and the solution is performed by entering the lightning time value in the FEM analysis. The electric transient solver does not provide adaptive meshing, so we will first solve using electrostatic solver and import its adaptively redefined mesh for use in the electric transient solution.

The electrostatic field simulator computes static electric fields due to potential differences and static electric charges. In Maxwell 3D, the fundamental unit of the finite element is a tetrahedron. The electric scalar potential inside each tetrahedron at each of the four vertices and at each of the six mid-edge nodes are determined by the solver. The potential value of each element is estimated with a 2 nd order quadratic equation.

Figure 1 shows a flow chart of the overall simulation process in Maxwell 3D. First, a three-dimensional module is opened and a solution method (Electrostatic, Electric Transient, etc.) is selected. The electrostatic problem is described by defining the geometry in the Cartesian coordinate system, material properties, voltages, boundary conditions. The geometry is directly imported in FEM Software. Unlike an electrostatic solution, the electric transient voltage curve is defined into the design data sets and the solution is performed by entering the lightning time value in the FEM analysis. Generated solutions are updated by using the adaptive analysis until the target percent error specified by the user, iteratively. Thus, a much more accurate solution is achieved by reducing the size of individual elements in the areas where the error is high. As the electric transient solver does not provide adaptive meshing, so we will first solve using electrostatic solver and import its adaptively redefined mesh for use in the electric transient solution.

Electrostatic solver computes the static electric fields resulting from voltages or from static charges distribution in a structure. For electrostatic solver; it is supposed that all objects are stationary. There is no time variation and current flow in conductors such that there is no electric field inside conductors.

The electrostatic field solver computes Poisson equation for the electric potential unknown, with appropriate boundary conditions:

$\nabla^{2} \varnothing=\rho / \varepsilon$

where $\varnothing$ is the electric potential, $\varepsilon$ is the permittivity, and $\rho$ is the charge density. Electrostatic solver computes the electric field using the following relationship:

$E=-\nabla \varnothing$

Equation (3) shows the connection between electric flux density $D$ and the electric field strength $E$. 


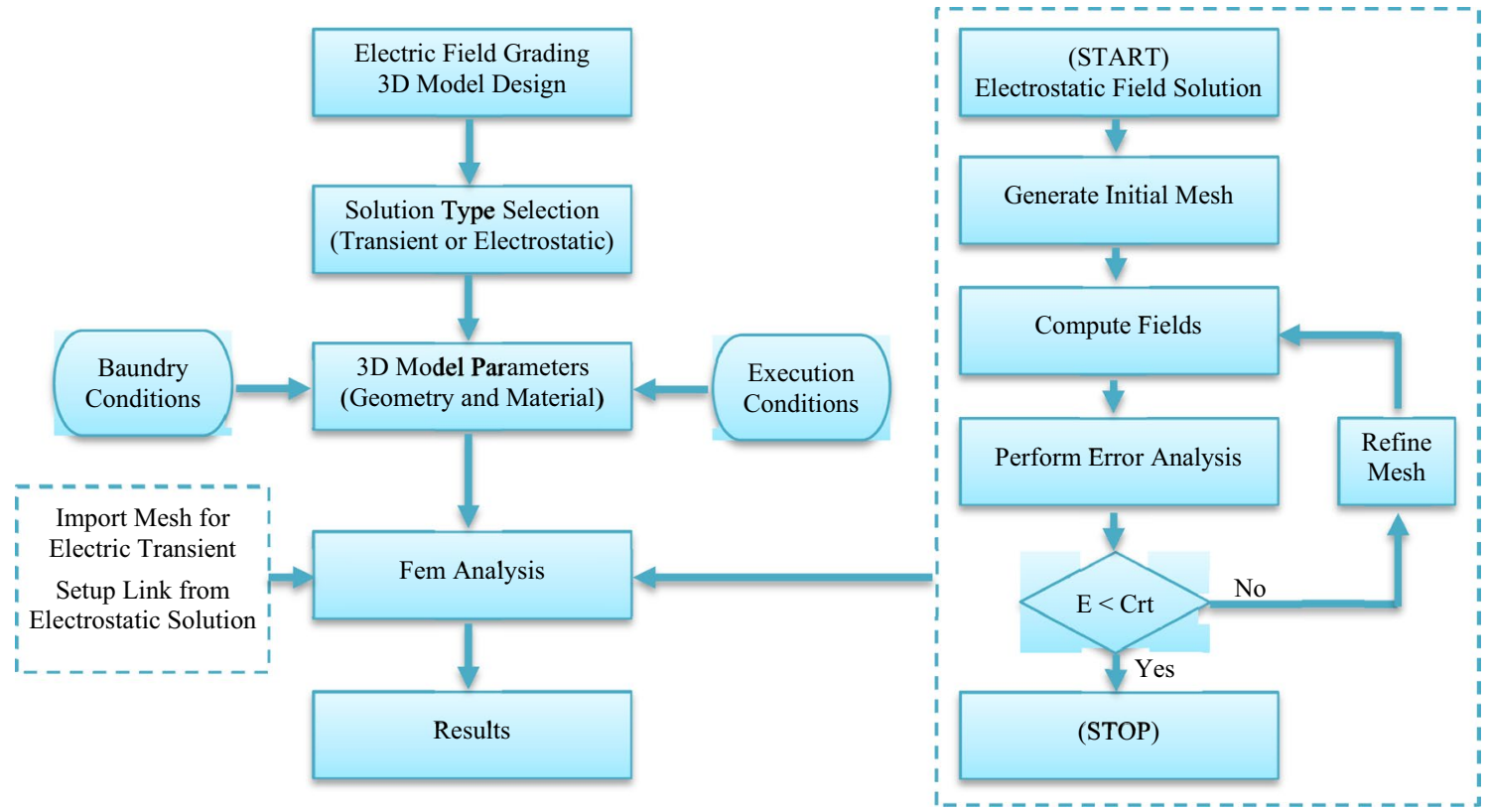

Fig. 1 The flow chart of the program

$D=\varepsilon E$

The energy, $W$ that is stored in a static electric field is given by the following equation:

$W=\frac{1}{2} \int_{v} \vec{E} \cdot \vec{D} d v$

The electric energy overall volume is computed by assuming that the electric field is uniform for a differential volume $(d v)$ :

$\mathrm{W}=\frac{1}{2} \int_{\mathrm{v}} \frac{1}{2} \varepsilon(-\nabla \varnothing) \mathrm{dv}$

Equation (5) can be rewritten in the Cartesian coordinate system as

$W=\iiint\left[\frac{1}{2}\left\{\varepsilon_{x}\left(\frac{d \varnothing}{d x}\right)^{2}+\varepsilon_{y}\left(\frac{d \varnothing}{d y}\right)^{2}+\varepsilon_{z}\left(\frac{d \varnothing}{d z}\right)^{2}\right\}\right] d x d y d z$

The electric transient solver is used to analyze the electric field distribution in objects subjected to time-varying potentials, e.g., impulse induced voltage, and overvoltage. Also, it applied to design insulator, lightning arrester, insulation constructions, and other applications that may involve conductive, semi-conductive, insulator, and anisotropic or nonlinear materials. The electric transient solver uses Eq. (9) to obtain ohmic loss, charge density and electric field values. The definition of the electric current density $\mathrm{J}$ is described as:
$J=\sigma E$

Using of the Eqs. (1), (2) and (7), the final Equation is obtained as:

$\nabla J+\frac{\partial \rho}{\partial t}=0$

$-\nabla(\sigma \nabla \varnothing)-\nabla\left(\varepsilon_{r} \varepsilon_{o} \nabla \frac{\partial \varnothing}{\partial t}\right)=0$

where $\rho$ is charge density, $\sigma$ is the conductivity.

\section{Analysis of field grading device}

The metal-enclosed switchgear consists of the cabinet made of stainless steel, a dielectric material made of porcelain, a fuse rod made of copper, an electric field grading device made of aluminium, a grounding material made of copper. The electrical properties of the materials used in the metal-enclosed switchgear are presented in Table 1.

The geometry of the measurement transformer, fuse and electric field grading devices are shown in Fig. 2 with the projections of the entire system on $x-y-z$ planes. In Fig. $2 c$, the distance between field grading devices is $0.35 \mathrm{~m}$. And the distance between the nearest side wall and the center of devices is taken as $0.29 \mathrm{~m}$. Investigations of the electric field distribution and transient characteristics of the grading devices have been made by applying 
Table 1 Material properties of the metal-enclosed switchgear

\begin{tabular}{llllc}
\hline Material & $\begin{array}{l}\text { Relative } \\
\text { permittivity } \\
(-)\end{array}$ & $\begin{array}{l}\text { Relative } \\
\text { permeability } \\
(-)\end{array}$ & $\begin{array}{l}\text { Bulk con- } \\
\text { ductivity } \\
(\mathrm{S} / \mathrm{m})\end{array}$ & $\begin{array}{l}\text { Mass } \\
\text { density } \\
\left(\mathrm{kg} / \mathrm{m}^{3}\right)\end{array}$ \\
\hline Porcelain & 5.7 & 1 & 0 & 2400 \\
Epoxy & 3.6 & 1 & 0 & 450 \\
Aluminium & - & 1.000021 & $38,000,000$ & 2689 \\
Copper & - & 0.999991 & $58,000,000$ & 8933 \\
Tin & - & 1 & $8,670,000$ & 7304 \\
Stainless & - & 1 & $1,100,000$ & 8055 \\
steel & & & & \\
\hline
\end{tabular}
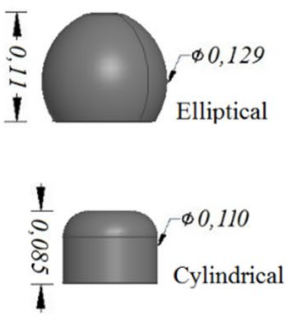

(a)

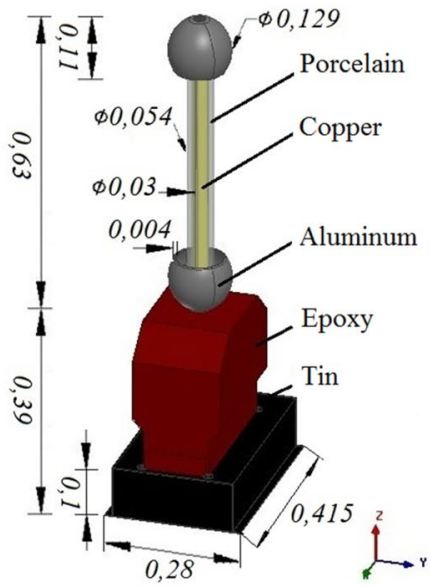

(b) the transient voltage of $170 \mathrm{kV}$ and the steady-state voltage of $36 \mathrm{kV}$.

\subsection{Electric transient analysis of the system}

Ansys Maxwell has a transient electrostatic solver. In order to simulate the impulse withstand voltage tests in the transient solution, the design datasets of the program are defined in accordance with the standard [24-26]. Impulse withstand voltage is known as short duration and singlepole wave shape $(1.2 / 50 \mu \mathrm{s})[26,27]$. As the electrical transient analysis takes a long time, the system is defined as a single-phase and analyzed separately. The cabinet is grounded. For both electrode structures, solutions have been implemented in the electric transient module in software by applying impulse voltage as defined in Fig. 3. The mesh structure of electrostatic analysis has been imported to the electric transient section in the program (setup by link). The total number of elements in the mesh is 848134

Fig. 2 Electric field grading devices (a), a single phase (b), the projections of the entire system (c)

Fig. 3 Impulse withstand voltage $1.2 / 50 \mu \mathrm{s}, 170 \mathrm{kV}$

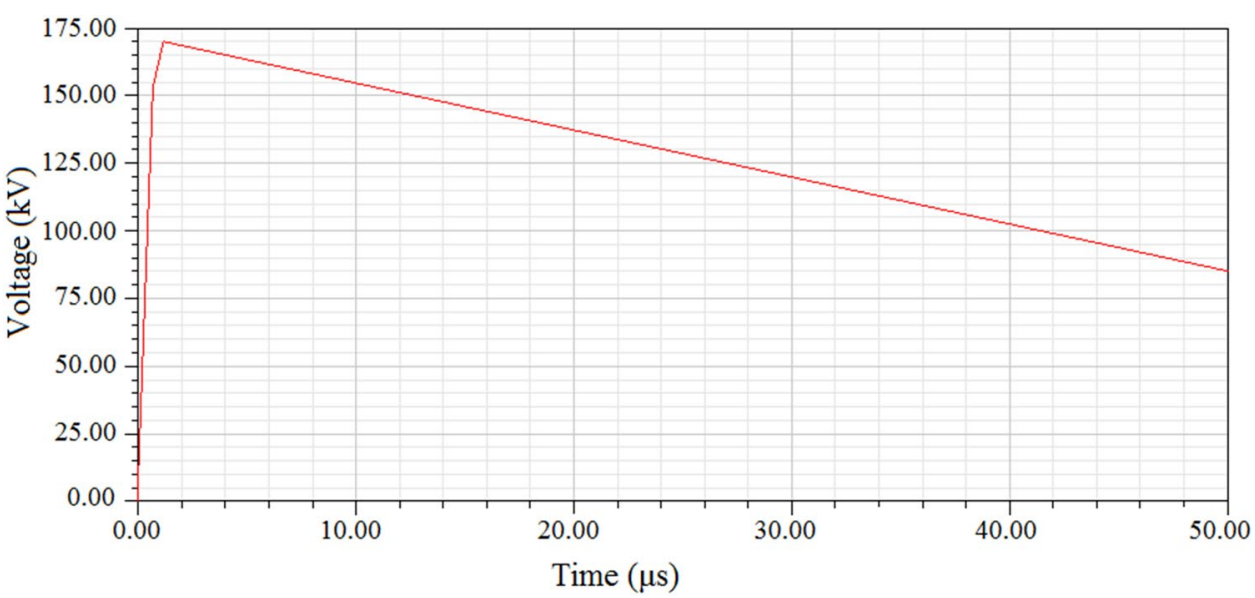


at electrostatic analysis. A computer with Intel i5 $2.8 \mathrm{GHz}$ processor and 6 GB RAM was used for system modeling. The elapsed time for the electrostatic field solution was $50 \mathrm{~min}$ and the time for the electric transient analysis was $615 \mathrm{~min}$. This analysis is a very important stage to find out which electric field grading device design is more appropriate.

The increase in the potential difference between the poles means that the energy increases for a single pole. Therefore, the energy in the system is expected to decrease or increase depending on the change in voltage. Total energy change in Fig. 4 shows that more energy is accumulated on the cylindrical electrode than the elliptical electrode. The ratio of both values has reached 1.5 times at the peak points $(0.468 \mu \mathrm{s})$ as shown in Fig. 4 . For this system, it can be said that the electric field grading device that has a fast energy change is more disadvantageous than the slow one.

Figure 5 shows the potential lines for both systems. It can be noticed that the potential lines on the epoxy
Fig. 4 The total energy change depending on the time

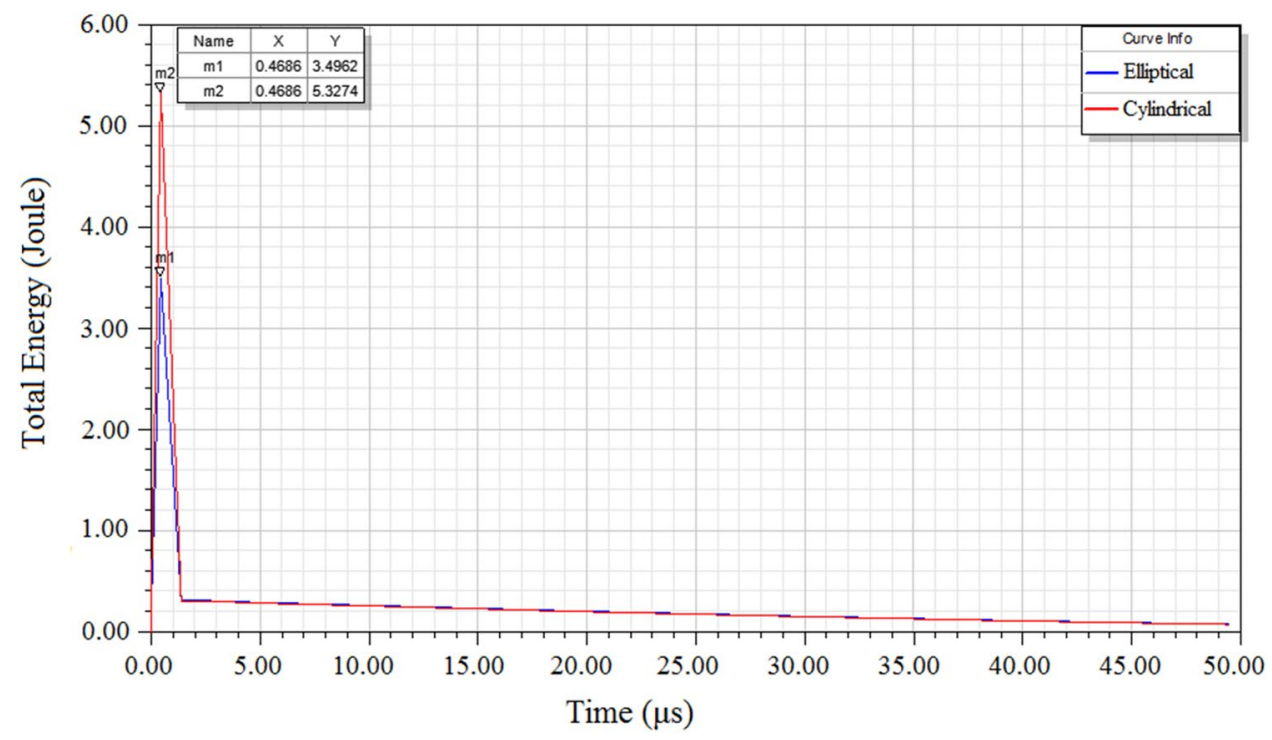

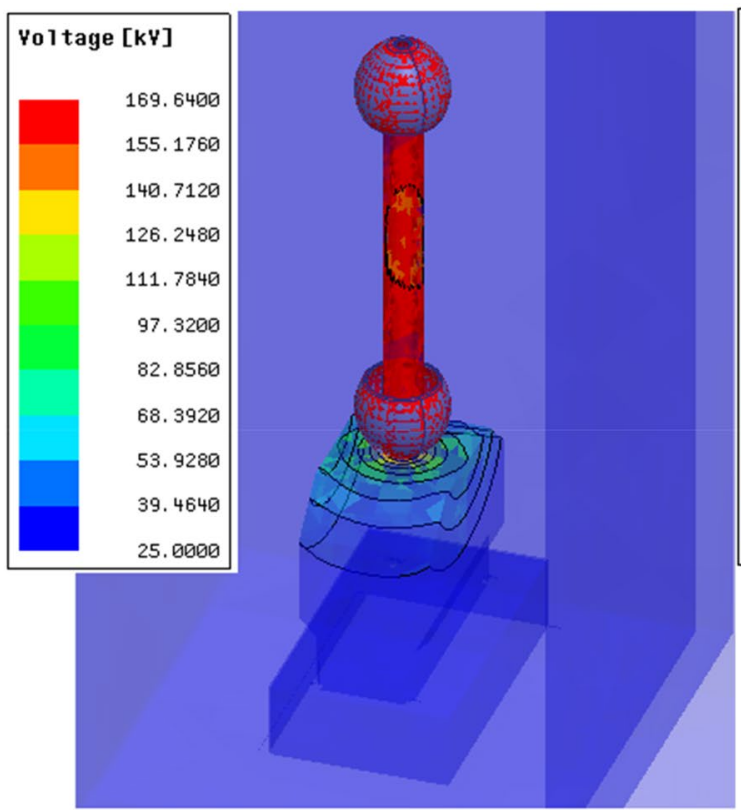

(a)

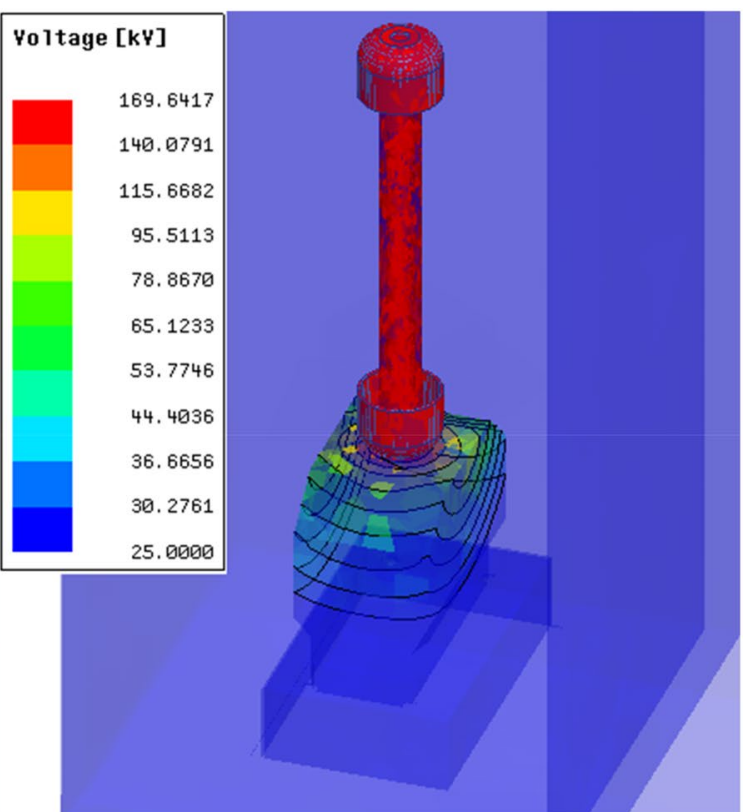

(b)

Fig. 5 Elliptical (a) and cylindrical (b) electric field grading device response to the impulse withstand voltage 
material belonging to the elliptic system are less than the cylindrical system. In some areas on the epoxy, potential varies about $44-53 \mathrm{kV}$ for the cylindrical system. It is clear that the elliptical electrode system is advantageous to electric field grading for both analysis results. Therefore, subsequent analysis and real system products have been carried out on the elliptic design basis.

For a more detailed analysis of the elliptic design, Fig. 6 should be examined. In order to determine the effect of elliptical electric field grading device to the electric field distribution, three numerical calculations have been performed: (1) only copper in the grounded cylinder; (2) with copper and porcelain in the grounded cylinder; (3) with copper, porcelain and elliptical aluminium grading device in the grounded cylinder.
As shown in Fig. 6a, the E-field in the inner zone of the fuse conductor is zero. In the case of porcelain insulation over the fuse conductor, leads to a reduction in the E-field. This is an expected result. The E-field grading device does not have much effect on the electric field change in the middle regions. The E-field distribution on the elliptical grading device mounted on the head part is slightly different as shown in Fig. 6b. The E-field intensity reduces from the end to the bottom. The resulting electric field, $\mathrm{E}$ is a non-uniform field because of system structures and increases near grading devices especially. Even though the electric field grading device by itself is symmetric, the symmetry is lost due to grounded structures that close to it in the cabin. Simulations showed that E-field intensity is affected by the shape of the field grading devices.

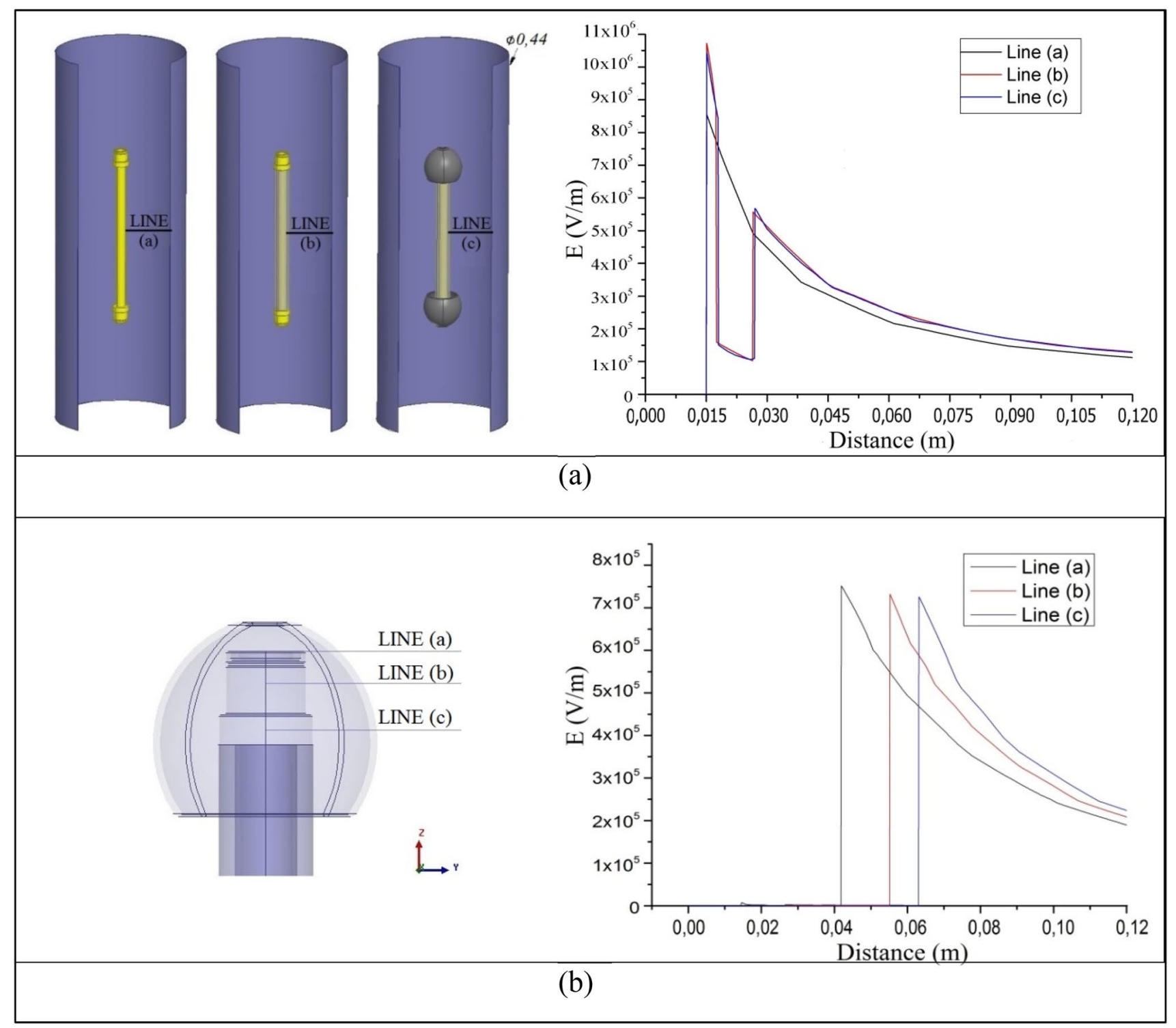

Fig. 6 The electric field change for a full shape, $\mathbf{b}$ only head 


\subsection{The steady-state analysis of the system}

Change in shape of grading device in the form of corners or edges in the cabinet leads to the concentration of $\mathrm{E}$ fields at such locations resulting in higher electric stresses on the dielectric $[27,28]$. Due to the long-term operation conditions, pollution, aging or design flaws, the structure of the grading device can no longer be homogeneous. Therefore, partial discharge initiation can be seen in such structures because of the uneven distribution of E-field strength. The steady-state analyses have been performed on the elliptic system as shown in Figs. 7 and 8. In order to make a steady-state solution, $36 \mathrm{kV}$ is applied to the middle copper with the electrodes while the cabinet and the other electrodes are defined to $0 \mathrm{kV}$. E-field vectors of middle part of the system are shown in Fig. 8.

The E-field vectors of the bottom and top field grading devices are not symmetrical. The main cause of the non-symmetry of the field lines is due to the behavior of the dielectric materials. The E-field $y-z$ and $x-z$ projections plot help to understand the critical regions in the metalenclosed switchgear as shown in Figs. 9 and 10. The E-field distribution on the cabinet is non-uniform and the maximum value of the E-field is $114,890 \mathrm{~V} / \mathrm{m}$ at the elliptical grading device edge on $y-z$ projections, as shown in Fig. 9. Therefore, no flashover or arc events are expected in the steady-state condition.

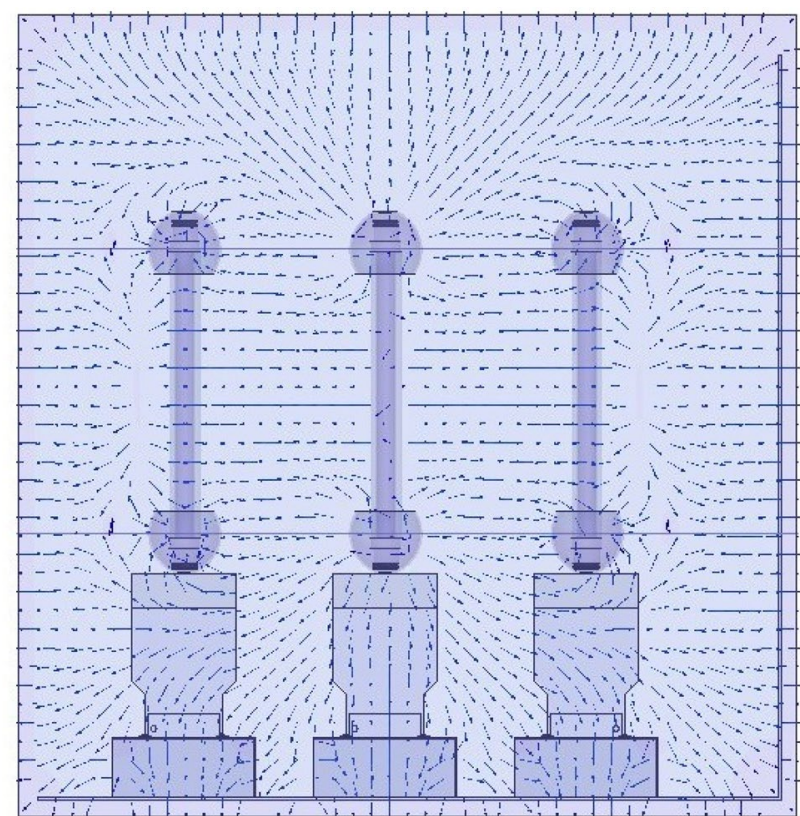

Fig. 8 The electric field vectors of the middle part of the system

\section{Laboratory tests and assessments}

In this section, the power frequency voltage withstand test and the impulse voltage test are carried out according
Fig. 7 Voltage distribution of the entire system

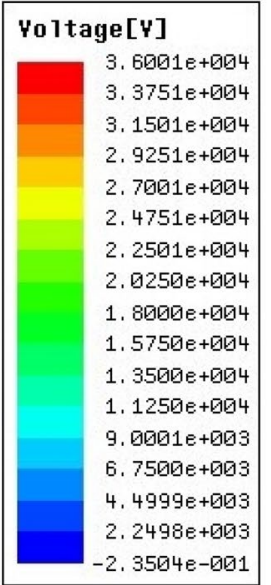




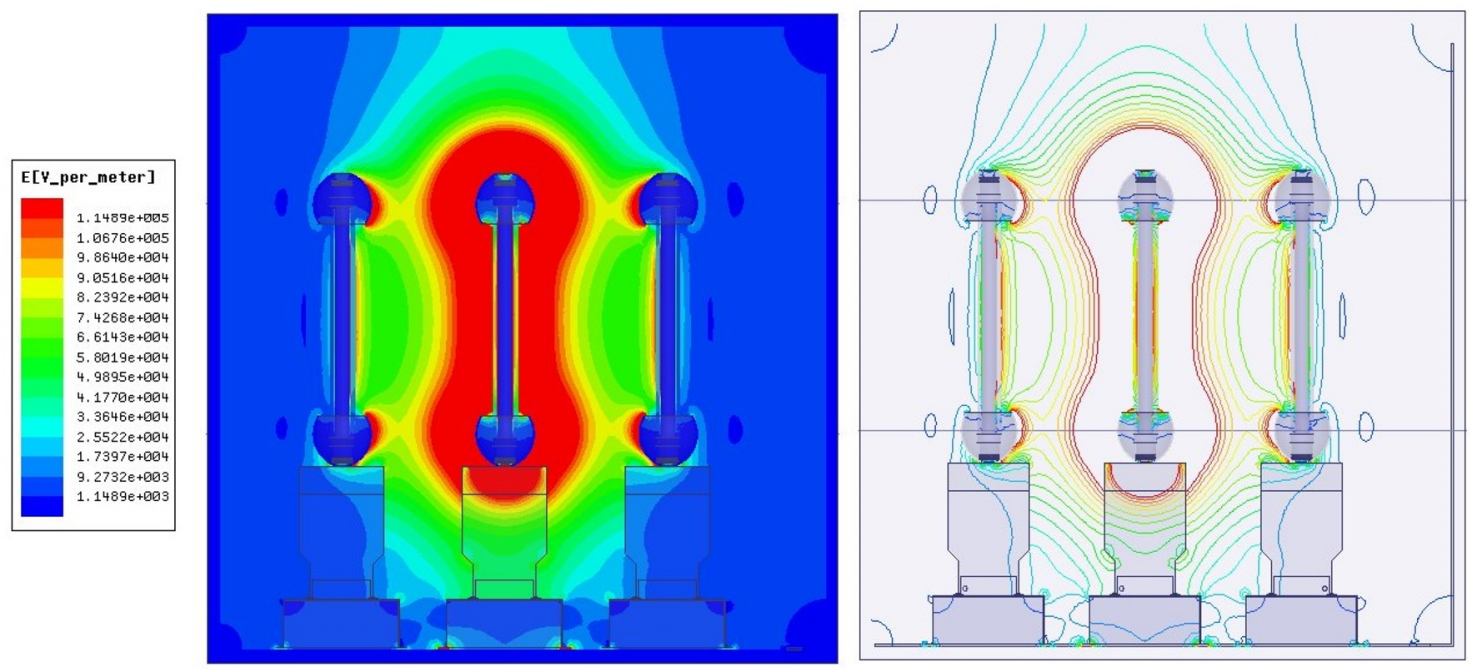

Fig. 9 The electric field of $y-z$ projections

Fig. 10 The electric field on $x-z$ projections

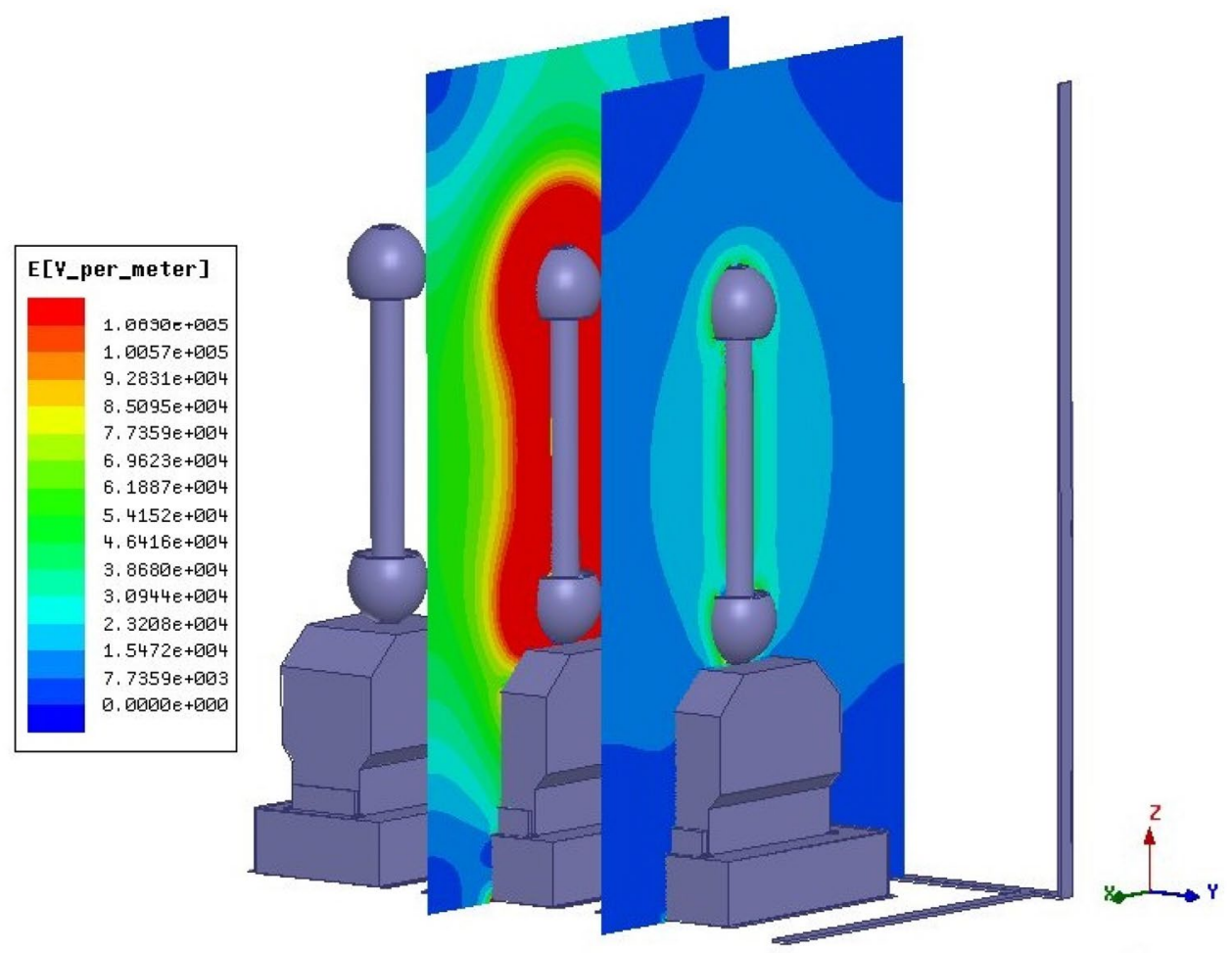

to IEC 62271-200 and IEC 60060-1 standards. These tests are applied to both old and proposed grading devices in metal-enclosed switchgear. The metal-enclosed switchgear and test parameters are presented in Table 2. Also, the physical conditions of the environment are very important for the accurate assessment of the test results. The values, temperature $\left(21^{\circ} \mathrm{C}\right)$, pressure $(689 \mathrm{mmHg})$ and relative humidity (61\%) are taken into consideration in the testing phase.

In the cabinet, the integrated electric field grading devices are tested to observe the impulse voltage withstand performance, experimentally. Both sides of the middle switch poles are permanently connected to the ground in the open position. Therefore, the test voltage is only 
Table 2 Metal-enclosed switchgear and test parameters

\begin{tabular}{lll}
\hline Technical definitions & Values & Unit \\
\hline Rated voltage & 36 & $\mathrm{kV}$ \\
Rated frequency & 50 & $\mathrm{~Hz}$ \\
$\begin{array}{l}\text { Rated power frequency withstand } \\
\text { voltage }\end{array}$ & $70(1)$ & $\mathrm{kV}(\mathrm{min})$ \\
Rated impulse withstand voltage & $170(1.2 / 50)$ & $\mathrm{kV}(\mu \mathrm{s})$ \\
Rated current & 630 & $\mathrm{~A}$ \\
Short-circuit breaking current & 16 & $\mathrm{kA}$ \\
Peak current withstand & 40 & $\mathrm{kA}$ \\
Protection class & $\mathrm{IP} 4 \mathrm{X}$ & - \\
Dimensions & $1000 / 1400 / 2250$ & $\mathrm{~mm}$ \\
Weight & 860 & $\mathrm{~kg}$ \\
\hline
\end{tabular}

applied to the middle phase. The manufactured metalenclosed switchgear parts are shown in Fig. 11.

The applied test voltages are determined by taking into consideration the atmospheric correction factors which are given in IEC 60060-1 standard. According to these assumptions, $\mathrm{U}$ is determined as $153 \mathrm{kV}$ approximately. In the test stage, A $1.2 / 50 \mu$ s impulse voltage with a $153 \mathrm{kV}$ peak is applied to the middle phase, while the other poles and cabinet are grounded. This step is repeated 15 times for each phase as positive and negative polarity. No breakdown or flashover occurred among devices and ground components during the test. The power frequency voltage withstands performance of integrated electric field grading devices are tested for each high voltage terminal in the cabinet, experimentally. This is the certain value of sinusoidal power frequency voltage in accordance with IEC 60060-1 as the factory routine test. In this stage, no breakdown or flashover occurred among devices and ground components during the test.

\section{Conclusion}

Analytical solutions can be applied to the known standard electrode structures. However, the use of analytical calculations will give erroneous results for the complex electrode geometries. Electric field analysis plays a critical role to optimize the form of the systems which have non-uniform complex structures. Ansys-Maxwell software provide an efficient way to the determination of the E-field distribution on the devices using numerical analysis. Within the scope of this study, a proposed electric field grading device that will be used in the metal-enclosed switchgear has been designed, and tested both in simulation and in the laboratory environment, respectively. The transient and steady-state analyses have been performed using the software tools to compare with the laboratory experiments.

The manufactured device is neither fully spherical nor fully cylindrical. The proposed design has an elliptical shape. The results show that the E-field on the electric field grading device could be reduced by enlarging the surface. However, enlarging the surface also refers to the proximity of electrodes to each other or to the cabinet

Fig. 11 Manufactured system

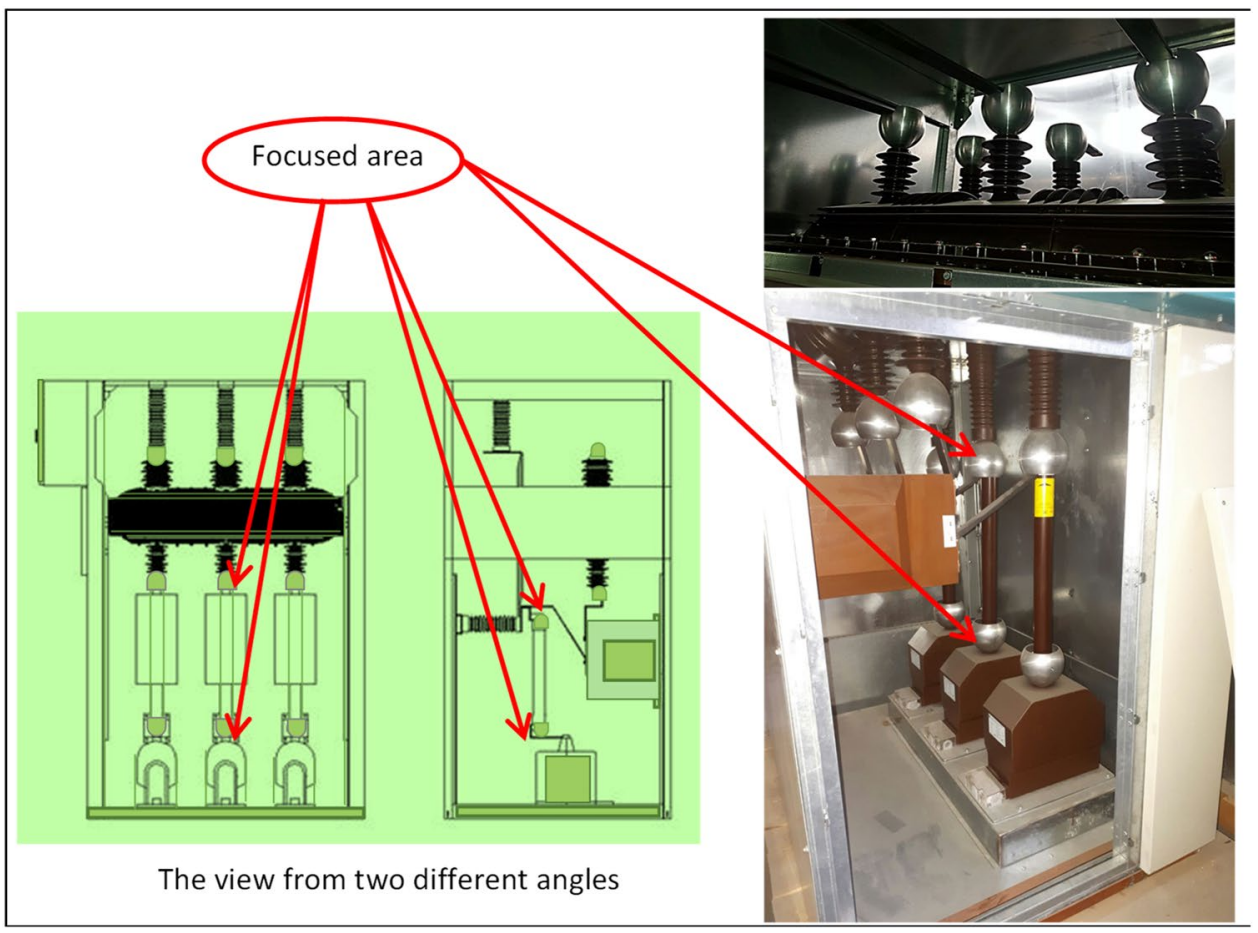


wall. The proximity in the electrode systems means the loss of homogeneity in the electric field. That is why an elliptical design is preferred in the system.

In metal-enclosed switchgear units, the structure used in these locations is generally cylindrical in the literature. In this study, the suitability of a different geometry structure has been tested. It is revealed that electric field distributions in the locality for the elliptical system are less than in the cylindrical system. Consequently, successful results have been achieved in the laboratory tests made according to the standards.

Acknowledgements The authors would like to thank the Turkish Electromechanical Industry Company (TEMSAN) for their financial support in design and HIZAL laboratory testing processes.

\section{Compliance with ethical standards}

Conflict of interest The authors declare that they have no known competing financial interests or personal relationships that could have appeared to influence the work reported in this paper.

Open Access This article is licensed under a Creative Commons Attribution 4.0 International License, which permits use, sharing, adaptation, distribution and reproduction in any medium or format, as long as you give appropriate credit to the original author(s) and the source, provide a link to the Creative Commons licence, and indicate if changes were made. The images or other third party material in this article are included in the article's Creative Commons licence, unless indicated otherwise in a credit line to the material. If material is not included in the article's Creative Commons licence and your intended use is not permitted by statutory regulation or exceeds the permitted use, you will need to obtain permission directly from the copyright holder. To view a copy of this licence, visit http://creativecommons .org/licenses/by/4.0/.

\section{References}

1. Phillips AJ et al (2014) Electric field limits for the design of grading rings for composite line insulators. IEEE Trans Power Deliv 30(3):1110-1118. https://doi.org/10.1109/TPWRD.2014.23620 74

2. Paredes-Olguin M et al (2013) Electric stress grading on bushings of combined instrument transformers using high permittivity polymeric composites. IEEE Trans Dielectr Electr Insul 20(6):2335-2342. https://doi.org/10.1109/EIC.2013.6554254

3. Christos $Z$ et al (2015) Development of electric-field stress control devices for a $132 \mathrm{kV}$ insulating cross-arm using finiteelement analysis. IEEE Trans Power Deliv 31(5):2105-2113. https ://doi.org/10.1109/TPWRD.2015.2499795

4. Natarajan M et al (2015) Analysis of stress control on 33-kV nonceramic insulators using finite-element method. Electr Power Compon Syst 43(5):566-577. https://doi.org/10.1080/15325 008.2014 .994242

5. Yundong C et al (2004) Investigation on insulation performance for medium voltage vacuum switch cabinet. In: XXIst International symposium on discharges and electrical insulation in vacuum proceedings, pp 660-663. https://doi.org/10.1109/ DEIV.2004.1422706
6. Domínguez DC et al (2013) Optimized design of electric field grading systems in $115 \mathrm{kV}$ non-ceramic insulators. IEEE Trans Dielectr Electr Insul 20(1):63-70. https://doi.org/10.1109/ TDEl.2013.6451342

7. Ilhan S, Ozdemir A (2011) $380 \mathrm{kV}$ corona ring optimization for AC voltages. IEEE Trans Dielectr Electr Insul 18(2):408-417. https ://doi.org/10.1109/TDEl.2011.5739444

8. Qin BL et al (1990) Efficient computation of electric field in high voltage equipment. IEEE Trans Magn 26(2):387-390. https://doi. org/10.1109/20.106335

9. Ashouri M, Mırzaı M, Gholamı A (2010) Calculation of voltage distribution along porcelain suspension insulators based on finite element method. Electr Power Compon Syst 38(7):820831. https://doi.org/10.1080/15325000903489694

10. Que W, Sebo SA (2002) Typical cases of electric field and voltage distribution calculations along polymer insulators under various wet surface conditions. In: IEEE Annual report conference on electrical insulation and dielectric phenomena, pp 840-843. https://doi.org/10.1109/CEIDP.2002.1048926

11. Nie $D$ et al (2013) Optimization design of grading ring and electrical field analysis of $800 \mathrm{kV}$ UHVDC wall wall bushing. IEEE Trans Dielectr Electr Insul 20(4):1361-1368. https://doi. org/10.1109/TDEl.2013.6571457

12. Ilhan S, Ozdemir A (2011) Effects of corona ring design upon impulse voltage withstand level of $380 \mathrm{kV}$ V-strings. IEEE Trans Dielectr Electr Insul 18(5):1638-1646. https://doi.org/10.1109/ TDEI.2011.6032834

13. Monga $S$ et al (2006) Design optimization of high voltage bushing using electric field computations. IEEE Trans Dielectr Electr Insul 13(6):1217-1224. https://doi.org/10.1109/TDEl.2006.25819 3

14. Doshi T et al (2011) Electric field computation of composite line insulators up to $1200 \mathrm{kV}$ AC. IEEE Trans Dielectr Electr Insul 18(3):861-867. https://doi.org/10.1109/TDEl.2011.5931075

15. Hrastnik J, Pihler J (2009) Designing a new post insulator using 3-D electric-field analysis. IEEE Trans Power Deliv 24(3):13771381. https://doi.org/10.1109/TPWRD.2009.2022662

16. Yanabu S, Zaima E, Hasegawa T (2006) Historical review of high voltage switchgear developments in the 20th century for power transmission and distribution system in Japan. IEEE Trans Power Delivery 21(2):659-664. https://doi.org/10.1109/TPWRD .2005 .861228

17. Kalkstein EW et al (1995) Safety benefits of arc-resistant metalclad medium-voltage switchgear. IEEE Trans Ind Appl 31(6):1402-1411. https://doi.org/10.1109/28.475733

18. Smajic J et al (2011) 3D full-Maxwell simulations of very fast transients in GIS. IEEE Trans Magn 47(5):1514-1517. https://doi. org/10.1109/CEFC.2010.5481807

19. Kumru C, Kocatepe C, Arıkan $O$ (2015) An investigation on electric field distribution around $380 \mathrm{kV}$ transmission line for various pylon models. Int J Electr Comput Energ Electron Commun Eng 9(8):138. https://doi.org/10.5281/zenodo.1108656

20. Kara A, Kalenderli Ö, Mardıkyan K (2006) Effect of dielectric barriers to the electric field of rod-plane air gap. In COMSOL users conference, pp 1-10

21. IEC Standard 62271-200 (2011) AC metal-enclosed switchgear and controlgear for rated voltages above $1 \mathrm{kV}$ and up to and including $52 \mathrm{kV}$. IEC Standard, Geneva

22. IEC Standard 60060-1 (2010) High voltage test techniquespart 1: general definitions and test requirements. IEC Standard, Geneva

23. Özkaya M (1996) High voltage technique. Birsen Publishing, Ankara (in Turkish)

24. Arora R, Mosch W (2011) High voltage and electrical insulation engineering. Wiley, Hoboken 
25. Kuffel J, Zaengl WS, Kuffel P (1984) High voltage engineering fundamentals. Pergamon, New York

26. Lucas JR (2001) High voltage engineering. Open University of Sri Lanka, Moratuwa

27. Wadhwa CL (2007) High voltage engineering. New Age International, Lincolnshire

28. Dedeoğlu S, Yilmaz S (2014) Design and implementation of a programmable high-voltage impulse measurement system. Turk
J Electr Eng Comput Sci 22(2):262-275. https://doi.org/10.3906/ elk-1205-82

Publisher's Note Springer Nature remains neutral with regard to jurisdictional claims in published maps and institutional affiliations. 